\title{
The efficacy of the novel zinc-containing desensitizer CAREDYNE Shield on dentin hypersensitivity: a study protocol for a pilot randomized controlled trial
}

Takashi Matsuura ${ }^{* \dagger}$ D, Megumi Mae ${ }^{\dagger}$, Masayuki Ohira, Yasunori Yamashita, Ayako Nakazono, Kouji Sugimoto, Kajiro Yanagiguchi and Shizuka Yamada

\begin{abstract}
Background: Dentin hypersensitivity $(\mathrm{DH})$ is a condition characterized by short and sharp episodes of pain which will arise in response to tactile, chemical, thermal, evaporative or osmotic stimuli. The painful symptoms cause discomfort in patients and reduce their quality of life. Recently, the novel zinc-containing desensitizer CAREDYNE Shield has been developed as a new type of desensitizer that acts by inducing chemical occlusion of dentinal tubules, and releasing zinc ion for root caries prevention. However, the clinical effectiveness of CAREDYNE Shield on $\mathrm{DH}$ remains unclear. Therefore, the aim of this study is to evaluate the effectiveness of CAREDYNE Shield on DH by comparing with that of another desensitizer, Nanoseal, commonly used in Japan.
\end{abstract}

Methods/design: This study protocol is a two-arm, parallel, pilot randomized controlled trial. Forty DH patients will be randomly allocated to two groups. Participants in the intervention group will be treated with CAREDYNE Shield, while those in the control group will be treated with Nanoseal. The primary outcome is the reduction of pain intensity in response to air stimuli measured with a 5-point verbal response scale from baseline to 4 weeks after the intervention, and Fisher's exact test will be used for analyses.

Discussion: CAREDYNE Shield can be casually applied to subgingival areas and proximal surfaces because it reacts with only tooth substance. Furthermore, zinc has been reported to reduce the demineralization of enamel and dentin and inhibit biofilm formation, plaque growth and dentin-collagen degradation. Therefore, CAREDYNE Shield may be expected to be a useful novel desensitizer that acts not only as a desensitizer but also as a root caries inhibitor.

Trial registration: UMIN Clinical Trials Registry (UMIN-CTR), ID: UMIN000038072. Registered on 21 September 2019.

Trial status: This study (protocol version number: version 1.4.0; approved on 22 October 2019) is ongoing. The recruitment of participants began in December 2019 and will be continued until November 2020 (Hanke, Am Dent Assoc 27:1379-1393, 1940).

Keywords: Dentin hypersensitivity, Desensitizer, CAREDYNE Shield, Nanoseal, Randomized clinical trial

\footnotetext{
* Correspondence: matsuurat@nagasaki-u.ac.jp

${ }^{\dagger}$ Takashi Matsuura and Megumi Mae contributed equally to this work. Department of Periodontology and Endodontology, Nagasaki University Graduate School of Biomedical Sciences, 852-8588, 1-7-1, Sakamoto, Nagasaki, Nagasaki, Japan
}

C C The Author(s). 2020 Open Access This article is licensed under a Creative Commons Attribution 4.0 International License, which permits use, sharing, adaptation, distribution and reproduction in any medium or format, as long as you give appropriate credit to the original author(s) and the source, provide a link to the Creative Commons licence, and indicate if changes were made. The images or other third party material in this article are included in the article's Creative Commons licence, unless indicated otherwise in a credit line to the material. If material is not included in the article's Creative Commons licence and your intended use is not permitted by statutory regulation or exceeds the permitted use, you will need to obtain permission directly from the copyright holder. To view a copy of this licence, visit http://creativecommons.org/licenses/by/4.0/. The Creative Commons Public Domain Dedication waiver (http://creativecommons.org/publicdomain/zero/1.0/) applies to the data made available in this article, unless otherwise stated in a credit line to the data. 


\section{Background}

Dentin hypersensitivity $(\mathrm{DH})$ is a condition characterized by short and sharp episodes of pain which arise in response to tactile, chemical, thermal, evaporative or osmotic stimuli, and which cannot be ascribed to any other form of dental defect or pathology [1, 2]. A recent systematic review reported the $\mathrm{DH}$ prevalence in various population to range from 1.3 to $92.1 \%$, and the estimated $\mathrm{DH}$ prevalence analyzed in a random-effects metaanalysis was 33.5\% (95\% confidence interval 30.2-36.7\%) [3]. The painful symptoms cause discomfort in patients and reduce their quality of life [4].

Normally, dentin is covered by enamel or cementum and is not affected by direct stimuli; however, once the dentin has been exposed and dentinal tubules are patent in the oral environment, the painful symptoms of $\mathrm{DH}$ arise in response to external stimuli. Dentin exposure results from a loss of enamel or cementum. The loss of enamel can be attributed to erosion, abrasion or abfraction, while the loss of cementum is caused by gingival recession associated with improper tooth-brushing, periodontal disease and periodontal surgery.

A number of theories have been suggested to explain the mechanisms of $\mathrm{DH}$; however, the hydrodynamic theory has been widely accepted $[5,6]$. According to this theory, fluid movement in the patent dentinal tubules occurs in response to external stimuli, stimulating sensory nerve endings located at the dentin-pulp interface. Thus, dentinal tubule occlusion, which reduces the fluid movement in the dentinal tubules, is one of the ideal $\mathrm{DH}$ treatments and is performed with adhesive systems or desensitizing agents that form insoluble mineral precipitates in the patent dentinal tubules [7].

One of desensitizing agents which form insoluble mineral precipitates in patent dentinal tubules is the fluoroaluminocalciumsilicate-based desensitizer Nanoseal (Nippon Shika Yakuhin, Yamaguchi, Japan). The components are similar to those of silicate cement. Nanoseal acts as a desensitizer by a chemical reaction resulting in insoluble nanoparticles that aggregate on the tooth surface for dentinal tubule occlusion and has been suggested to protect the root surface from demineralization via ions, such as calcium or fluorine, released from Nanoseal [8-10]. Thus, Nanoseal may act not only as a desensitizer but also as a root caries inhibitor.

Recently, the fluorozinccalsiumsilicate-based desensitizer CAREDYNE Shield (GC Dental Industrial Corporation, Tokyo, Japan) has been developed. It acts as a desensitizer by inducing chemical occlusion of dentinal tubules and contains a novel functional filler that releases not only ions, such as calcium and fluorine, but also zinc. Zinc has been reported to reduce the demineralization of enamel and dentin, and inhibit dentin collagen degradation, plaque growth and biofilm formation $[11,12]$.
However, the effectiveness of CAREDYNE Shield on $\mathrm{DH}$ still remains unclear; therefore, the aim of this study is to investigate the effectiveness of CAREDYNE Shield on DH by comparing with that of Nanoseal. The PICO question of this study is described in Table 1.

\section{Methods/design}

\section{Trial design}

This study protocol is a two-arm, parallel, pilot randomized controlled trial; that was developed in accordance with the Standard Protocol Items: Recommendations for Interventional Trials (SPIRIT) and Consolidated Standards of Reporting Trials (CONSORT) guidelines [13, 14]. A SPIRIT Checklist is attached in Additional file 1.

\section{Participant timeline}

To describe the time schedule of enrollment, intervention and assessment, a SPIRIT Figure and a CONSORT flow chart are presented in Table 2 and Fig. 1.

\section{Study setting}

All procedures of this study will be performed at the Department of Periodontology and Endodontology, Nagasaki University Graduate School of Biomedical Sciences, Nagasaki, Japan.

\section{Sample size}

No previous clinical studies have investigated the effectiveness of CAREDYNE Shield on DH; therefore, at least 15 participants will be required in each group to perform a sample size calculation in a subsequent study [15]. With a $20 \%$ dropout rate, a total of 40 participants will be recruited in this study.

\section{Eligibility screen}

When patients present with DH complaints at Nagasaki University Hospital, clinical diagnosis will be performed by the dentist in charge of the patient. Short and sharp pain episodes of pain which arise in response to air stimuli or tactile stimuli will be assessed with a three-way dental syringe or a dental explorer, caries or restoration will be assessed with a dental mirror and a dental explorer, severe periodontal diseases will be assessed with a dental mirror and a periodontal probe and the information about medical/dental history will be obtained by

\begin{tabular}{ll} 
Table 1 PICO question \\
\hline Criteria & Description \\
\hline P (participants) & Non-carious human permanent teeth with DH \\
I (Intervention) & DH treatment with CAREDYNE Shield \\
C (Control) & DH treatment with Nanoseal \\
O (Outcome) & The reduction of pain level in response to air stimuli
\end{tabular}

$\mathrm{DH}$ dentin hypersensitivity 
Table 2 Standard Protocol Items: Recommendations for Interventional Trials (SPIRIT) Figure

\begin{tabular}{lll}
\hline & Enrollment & Post allocation \\
\hline Time point & 0 & 4 weeks (range 3-5 weeks) \\
Enrollment: & & \\
$\quad$ Eligibility screen & $X$ & \\
Informed consent & $X$ & \\
Allocation & $X$ & \\
Intervention: & & \\
CAREDYNE Shield & $X$ & \\
Nanoseal & $X$ & \\
Clinical assessment: & & \\
Air blow & $X$ & $X$ \\
Inspection & $X$ & $X$ \\
Palpation & $X$ & \\
\hline
\end{tabular}

interview. Then, the eligibility criteria described in Table 3 will be verified.

\section{Informed consent}

After eligibility screen, the potential participants who met the eligibility criteria will be asked for their informed consent using the informed consent form. They will be then enrolled in this study by an examiner blinded to the allocation. A signed informed consent form is mandatory for enrollment.
Table 3 eligibility criteria

Inclusion criteria
Outpatients
Participants who presented with a DH complaint
Participants who agreed to participate in this study after
providing their informed consent

\section{Exclusion criteria}

Participants who have an allergy to the desensitizing materials used in this study

Participants who are pregnant or lactating

Participants who have undergone $\mathrm{DH}$ treatment within the last 6 months

Participants with systemic diseases that might influence the results of this study

Participants who present with pain complaints that might influence the results of this study

$\mathrm{DH}$ teeth with restoration that might influence the results of this study

$\mathrm{DH}$ teeth with caries or advanced periodontal disease

$\mathrm{DH}$ teeth that have undergone periodontal surgery or orthodontic treatment within the last 3 months

$\mathrm{DH}$ dentin hypersensitivity

\section{Randomized allocation}

After enrollment, baseline assessments will be performed by the examiner who enrolled the participant in this study. Randomized allocation will then be performed in a 1:1 ratio by the dentist in charge of the patient with

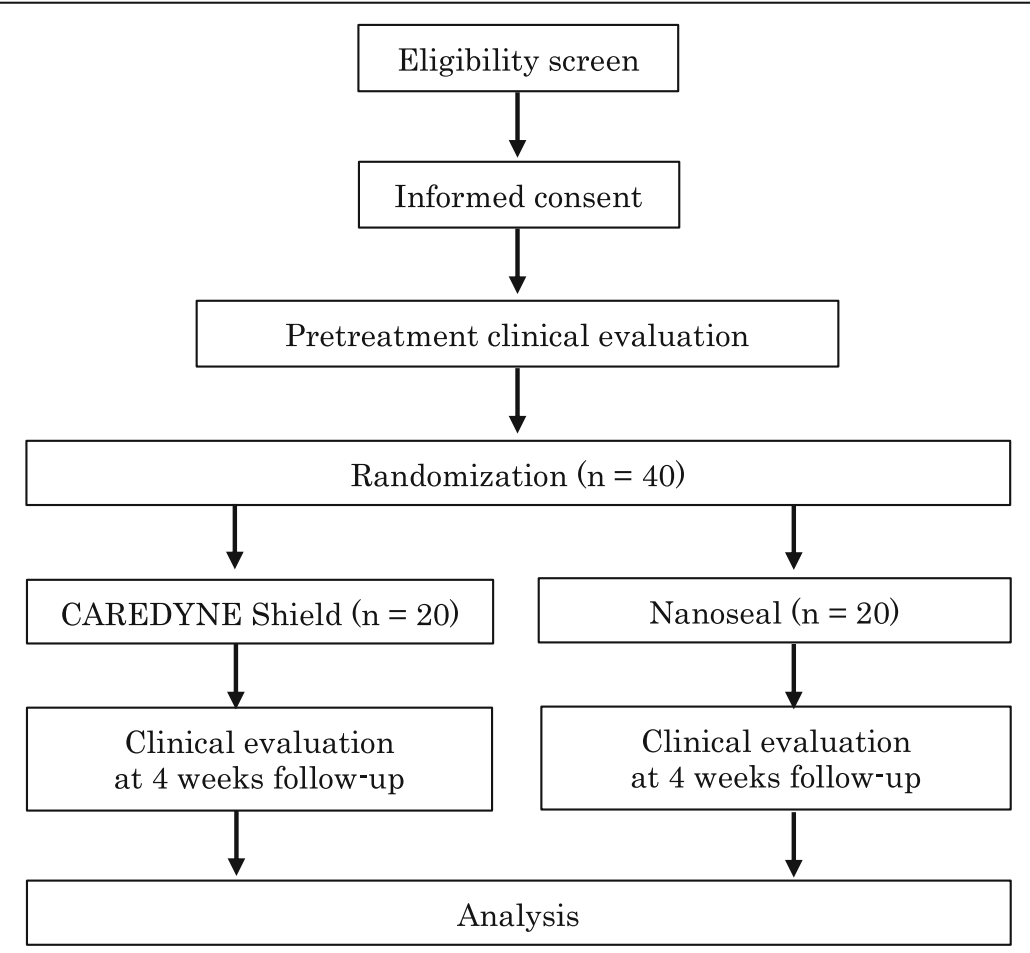

Fig. 1 Consolidated Standards of Reporting Trials (CONSORT) flow chart 
opaque, sealed envelopes prepared before participant recruitment and on which "Nanoseal" or "CAREDYNE Shield" is printed. Participants allocated to the intervention group will be treated with CAREDYNE Shield, while those allocated to the control group will be treated with Nanoseal. The information on the desensitizers used in this study is described in Table 4.

\section{Allocation concealment}

After the allocation, the operator who performed allocation will record the operator's name, baseline date and type of teeth to be treated in the allocation list. The list will not contain the allocated group name in order to conceal the allocation as well as the participant's name and ID number for personal information protection. Allocation information will be recorded in the electronic medical record system which research staff cannot check without leaving a record of their browsing history.

\section{Blinding of participants}

Participants will be blinded during this study and disclosure of allocation to participants will be performed in the following cases: (1) participant requests to stop or change the allocated intervention, (2) worsening disease or new disease occurs, (3) continuing this study is judged to be inappropriate for the participant or (4) this study is terminated.

\section{Intervention}

After randomized allocation, the participants in the intervention group will be treated with CAREDYNE Shield, and those in the control group will be treated with Nanoseal by the operator who performed allocation. Prior to the application of CAREDYNE Shield or Nanoseal, dental prophylaxis and water rinse will be performed to remove plaque deposits; the areas to be treated will be isolated with cotton rolls and dried with cotton pellets; two equal proportions of solution $A$ and solution B will be mixed with a micro-brush and applied to the dentin surface for $20 \mathrm{~s}$, followed by rinsing with water. Four weeks after the treatment, clinical assessments will be performed by the examiner who enrolled the participant in this study.

During this study, any other dental treatment to $\mathrm{DH}$ teeth are prohibited, and all intervention procedures will be recorded by the operator in an electronic chart to improve adherence to intervention protocols. Discontinuing intervention will be performed in the following case: (1) participant requests to stop or change the allocated intervention, (2) worsening disease or new disease occurs or (3) continuing this study is judged to be inappropriate.

\section{Primary outcome}

The primary outcome is the reduction of pain intensity in response to air stimuli measured with a 5-point verbal rating scale (VRS) from baseline to 4 weeks after the intervention. To evaluate the pain level, an air blast will be applied using a three-way dental syringe after the isolation of the DH teeth with cotton rolls, participants will then be asked verbally to rate the level of pain intensity using a 5-point VRS (numerical scale from 0 to 4 summarized in Table 5).

\section{Secondary outcome}

Secondary outcomes are the change in the gingival condition near the treated area measured with the Gingival Index (GI) and the change in the oral hygiene status at the treated dentin surface measured with the Plaque Index (PI) from baseline to 4 weeks after the intervention $[16,17]$. The GI and PI will be evaluated with inspection and palpation.

\section{Data collection}

Outcomes will be assessed at baseline and 4 weeks after the intervention by the examiner who enrolled the participant in this study. Calibration was performed to promote the data quality. The examiner's name, date of assessment, type of teeth and the acquired outcomes will be recorded in the assessment form and it will be given to the lead principal investigator.

\section{Data management}

Double data entry will be performed by two research staff independently. To ensure confidentiality, all documents obtained in this study will be kept strictly in a lockable filing cabinet in the office for at least 5 years after this study, before being destroyed using a shredder and discarded.

\section{Statistical design}

The primary outcome will be analyzed with Fisher's exact test according to the intention-to-treat principle. Participants who discontinue or deviate from the

Table 4 Desensitizers used in this study

\begin{tabular}{|c|c|c|}
\hline Material & Manufacturer & Composition \\
\hline CAREDYNE Shield & GC Dental Industrial Corporation, Tokyo, Japan & $\begin{array}{l}\text { Solution A: fluorozinccalciumsilicate glass } \\
\text { Solution B: 10-15\% phosphoric acid }\end{array}$ \\
\hline Nanoseal & Nippon Shika Yakuhin Co., Ltd., Shimonoseki, Japan & $\begin{array}{l}\text { Solution A: fluoroaluminocalciumsilicate glass } \\
\text { Solution B: } 10 \% \text { phosphoric acid }\end{array}$ \\
\hline
\end{tabular}


Table 5 Verbal rating scale

\begin{tabular}{ll}
\hline Score & Level of pain intensity \\
\hline 0 & No pain \\
1 & Mild pain \\
2 & Moderate pain \\
3 & Severe pain \\
4 & Extremely intense pain \\
\hline
\end{tabular}

intervention protocols or with any missing data will be excluded. No additional analyses will be performed.

\section{Access to data}

The principal investigator will have access to the final study data and make the final decision to terminate this study. No other research staff will have access to any data acquired in this study.

\section{Monitoring}

Monitoring will be performed by one of our research staff according to the standard operating procedures and the results will be given to the principal investigator within 2 weeks after monitoring. When adverse events or other unintended effects of the intervention happen, the principal investigator will perform appropriate treatments for the participant, report the incident to the Nagasaki University Hospital Clinical Research Ethics Committee (REC) and share this information with the research staff. A data monitoring committee is not necessary because DH treatment with Nanoseal or CAREDYNE Shield is general practice and a low-invasive intervention procedure.

\section{Potential benefits and harms}

This study will contribute to future clinical improvements. However, atopic dermatitis is a potential side effect of the intervention.

\section{Discussion}

Several limitations associated with the present study warrant mention. First, blinding of operators will not be performed; therefore, the risk of bias in this domain may be unavoidable. Second, the data will be collected just after the participant's training. Thus, VRS which is easier to understand for participants than a visual analog scale (VAS) will be performed; however, VRS is considered to be less sensitive than VAS [18, 19]. Third, it has been recommended that at least three different stimuli, specifically tactile, cold and air stimuli, should be used for clinical assessments of $\mathrm{DH}$ because $\mathrm{DH}$ may differ among stimuli [2]. However, only air stimuli will be evaluated in the present study because this study is being performed for sample size calculations, which is based on the primary outcome: the reduction of pain intensity in response to air stimuli. Tactile stimuli will be evaluated as a secondary outcome in a future, large-scale study. The GI and PI will be used as secondary outcomes in the present study. If a reduction in either is observed, the effectiveness of CAREDYNE Shield for the reduction of the GI and PI will be investigated in a future clinical study.

Desensitizers like CAREDYNE Shield and Nanoseal that induce chemical occlusion of dentinal tubules are biocompatible and react only with tooth substance; they can, therefore, be casually applied to subgingival areas and proximal surfaces. Furthermore, for several decades, in-vitro studies have investigated the function of zinc and reported that zinc reduced the demineralization of enamel and dentin and inhibited dentin collagen degradation, plaque growth and biofilm formation [20-24]. Thus, CAREDYNE Shield, which releases zinc ions, may be a useful novel desensitizer that acts not only as a desensitizer but also as a root caries inhibitor.

\section{Supplementary information}

Supplementary information accompanies this paper at https://doi.org/10. 1186/s13063-020-04426-8.

Additional file 1. Standard Protocol Items: Recommendations for Interventional Trials (SPIRIT) 2013 Checklist: recommended items to address in a clinical trial protocol and related documents.

\section{Abbreviations}

CONSORT: Consolidated Standards of Reporting Trials; DH: Dentin hypersensitivity; RCT: Randomized clinical trial; REC: Research Ethics Committee; SPIRIT: Standard Protocol Items: Recommendations for Interventional Trials; UMIN-CTR: The University Hospital Medical Information Network-Clinical Trials Registry

\section{Acknowledgements}

We thank Hayato Yokohara, Keisuke Ikushima and Daizaburo Mori of GC Dental Industrial Corporation, Tokyo, Japan for explaining about the characteristics and function of CAREDYNE Shield based on unpublished experimental data and providing the Nanoseal and CAREDYNE Shield.

\section{Authors' contributions}

TM is the main author of this manuscript and responsible for this study. All authors revised the manuscript and approved the final version of the manuscript.

\section{Funding}

This work was supported by JSPS KAKENHI, Grant Number JP19K24100.

\section{Availability of data and materials \\ Not applicable}

\section{Ethics approval and consent to participate}

This study protocol was approved by the Nagasaki University Hospital Clinical Research Ethics Committee (authorization number: 19102101) prior to participant recruitment, and registered with the University Hospital Medical Information Network-Clinical Trials Registry (UMIN-CTR; No. UMIN000038072) on 21 September 2019. Informed consent will be obtained from all study participants. The modified study protocol will not be performed without REC approval. The results of this study will be disseminated to the public via open-access publication. 


\section{Consent for publication}

Not applicable

\section{Competing interests}

TM received Nanoseal and CAREDYNE Shield from GC for this study, but the authors have not received any financial support. Any competing interests will be managed by the Conflict of Interest Committee of Nagasaki University Hospital.

Received: 11 January 2020 Accepted: 16 May 2020

Published online: 03 June 2020

\section{References}

1. Addy M, Dowell P. Dentine hypersensitivity - a review. Clinical and in vitro evaluation of treatment agents. J Clin Periodontol. 1983;10:351-63.

2. Holland GR, Narhi MN, Addy M, Gangarosa L, Orchardson R. Guidelines for the design and conduct of clinical trials on dentine hypersensitivity. J Clin Periodontol. 1997;24:808-13.

3. Favaro Zeola L, Soares PV, Cunha-Cruz J. Prevalence of dentin hypersensitivity: systematic review and meta-analysis. J Dent. 2019;81:1-6.

4. Bekes K, John MT, Schaller HG, Hirsch C. Oral health-related quality of life in patients seeking care for dentin hypersensitivity. J Oral Rehabil. 2009;36:45-51.

5. West NX, Lussi A, Seong J, Hellwig E. Dentin hypersensitivity: pain mechanisms and aetiology of exposed cervical dentin. Clin Oral Investig. 2013;17(Suppl 1):S9-19.

6. Brannstrom $M$, Linden $L A$, Astrom $A$. The hydrodynamics of the dental tubule and of pulp fluid. A discussion of its significance in relation to dentinal sensitivity. Caries Res. 1967;1:310-7.

7. Brannstrom M, Johnson G, Nordenvall KJ. Transmission and control of dentinal pain: resin impregnation for the desensitization of dentin. J Am Dent Assoc. 1979:99:612-8.

8. Lodha E, Hamba H, Nakashima S, Sadr A, Nikaido T, Tagami J. Effect of different desensitizers on inhibition of bovine dentin demineralization: micro-computed tomography assessment. Eur J Oral Sci. 2014;122:404-10.

9. Miyajima H, Ishimoto T, Ma S, Chen J, Nakano T, Imazato S. In vitro assessment of a calcium-fluoroaluminosilicate glass-based desensitizer for the prevention of root surface demineralization. Dent Mater J. 2016;35:399-407.

10. Mehta D, Gowda VS, Santosh A, Finger WJ, Sasaki K. Randomized controlled clinical trial on the efficacy of dentin desensitizing agents. Acta Odontol Scand. 2014;72:936-41.

11. Hasegawa T, Takenaka S, Ohsumi T, Ida T, Ohshima H, Terao Y, et al. Effect of a novel glass ionomer cement containing fluoro-zinc-silicate fillers on biofilm formation and dentin ion incorporation. Clin Oral Investig. 2019;24: 963-70.

12. Saad A, Nikaido T, Abdou A, Matin K, Burrow MF, Tagami J. Inhibitory effect of zinc-containing desensitizer on bacterial biofilm formation and root dentin demineralization. Dent Mater J. 2019;38(6):940-6.

13. Chan AW, Tetzlaff JM, Gotzsche PC, Altman DG, Mann H, Berlin JA, et al. SPIRIT 2013 explanation and elaboration: guidance for protocols of clinical trials. BMJ (Clinical research ed). 2013;346:e7586.

14. Schulz KF, Altman DG, Moher D. CONSORT 2010 Statement: updated guidelines for reporting parallel group randomised trials. BMJ (Clinical research ed). 2010;340:c332.

15. Lancaster GA, Dodd S, Williamson PR. Design and analysis of pilot studies: recommendations for good practice. J Eval Clin Pract. 2004;10:307-12.

16. Silness J, Loe H. Periodontal disease in pregnancy. II. correlation between oral hygiene and periodontal condition. Acta Odontol Scand. 1964;22:121-35.

17. Loe H, Silness J. Periodontal disease in pregnancy. I. prevalence and severity. Acta Odontol Scand. 1963;21:533-51.

18. Clark P, Lavielle P, Martinez H. Learning from pain scales: patient perspective. J Rheumatol. 2003;30:1584-8.

19. Breivik EK, Bjornsson GA, Skovlund E. A comparison of pain rating scales by sampling from clinical trial data. Clin J Pain. 2000;16:22-8.

20. Hanke MT. Studies on the local factors in dental caries. I. destruction of plaques and retardation of bacterial growth in the oral cavity. J Am Dent Assoc. 1940;27:1379-93.

21. Mohammed NR, Mneimne M, Hill RG, Al-Jawad M, Lynch RJ, Anderson P. Physical chemical effects of zinc on in vitro enamel demineralization. J Dent. 2014;42:1096-104.

22. Takatsuka T, Tanaka K, lijima Y. Inhibition of dentine demineralization by zinc oxide: in vitro and in situ studies. Dent Mater. 2005;21:1170-7.
23. Toledano M, Yamauti M, Osorio E, Osorio R. Zinc-inhibited MMP-mediated collagen degradation after different dentine demineralization procedures. Caries Res. 2012:46:201-7.

24. Saxton CA, Harrap GJ, Lloyd AM. The effect of dentifrices containing zinc citrate on plaque growth and oral zinc levels. J Clin Periodontol. 1986;13: $301-6$.

\section{Publisher's Note}

Springer Nature remains neutral with regard to jurisdictional claims in published maps and institutional affiliations.
Ready to submit your research? Choose BMC and benefit from:

- fast, convenient online submission

- thorough peer review by experienced researchers in your field

- rapid publication on acceptance

- support for research data, including large and complex data types

- gold Open Access which fosters wider collaboration and increased citations

- maximum visibility for your research: over $100 \mathrm{M}$ website views per year

At BMC, research is always in progress.

Learn more biomedcentral.com/submissions 